\section{Variância intrapessoal da ingestão de energia e nutrientes em adolescentes: correção de dados em estudos epidemiológicos}

\section{Within-person variance of the energy and nutrient intake in adolescents: data adjustment in epidemiological studies}

\section{Resumo}

Objetivo: Este artigo objetiva fornecer valores de componentes de variância intrapessoal da ingestão de energia e nutrientes que possibilita estimar a ingestão habitual em adolescentes, em inquéritos dietéticos quando há disponível somente um recordatório de 24 horas para cada indivíduo. Também será fornecido um exemplo de sua aplicação na correção da distribuição da ingestão de nutrientes proveniente de adolescentes de outro estudo. Métodos: Foram utilizados recordatórios de 24 horas de 215 adolescentes do município de São Paulo. Os componentes de variância foram estimados utilizando o Software for Intake Distribution Estimation (PC-SIDE). Para cada nutriente foi construído um modelo, estratificado por sexo, que inclui o dia e o mês da coleta, e variáveis de ponderação. Resultados: Os componentes de variância intrapessoal variaram de 0,55 para gordura mono-saturada (sexo feminino) a 0,96 para o folato (sexo masculino). Entre os macronutrientes e energia foram observados valores mais elevados para o sexo masculino. No exemplo da aplicação do componente de variância, foi estimada a distribuição da ingestão de vitamina $\mathrm{B} 6$ com menos viés comparada à distribuição sem a correção. Conclusão: Estes dados podem ser utilizados na estimativa da ingestão habitual de energia e nutrientes e cálculo da prevalência de inadequação em adolescentes de populações semelhantes, quando há disponível somente um recordatório de 24 horas para cada indivíduo do estudo.

Palavras-chave: Nutrientes. Adolescente. Inquérito sobre dietas. Avaliação nutricional. Consumo de energia. Métodos. do Estado do Rio de Janeiro - UERJ.

" Departamento de Epidemiologia da Faculdade de Saúde Pública a

Universidade de São Paulo-USP.

I" Departamento de Nutrição da Faculdade de Saúde Pública da Universidade de São Paulo - USP.
Este trabalho recebeu financiamento da Fundação de Apoio à Pesquisa do Estado de São Paulo FAPESP, processo n. 07/51489-9.

Correspondência: Eliseu Verly Jr. Departamento de Epidemiologia, Instituto de Medicina Social, Universidade do Estado do Rio de Janeiro. Rua São Francisco Xavier 524, Maracanã, Rio de Janeiro-RJ, 20550-900. E-mail: verlyjr@ims.uerj.br 


\section{Abstract}

Objective: The aim of this paper is to calculate within-person variance component of the energy and nutrient intake that can be useful to adjust intake distribution from adolescents when is available only a single 24-hour recall for each participant. It will also give an example of its use in the intake distribution adjustment in adolescents from another sample. Methods: It was used 24 hour recall from 215 adolescents living in São Paulo, Brazil. The variance components were estimated using the Software for Intake Distribution Estimation (PC-SIDE). It was made a model for each nutrient and sex of which were included covariates for day and month of intake and weight variable as well. Results: The within-person variance component ranged from 0.55 for mono saturated fat (female) up to 0.96 for folate (male). I was found higher values regarding to macronutrient and energy for males. In the example of the use of the variance component, it was estimated the distribution of intake of vitamin B6 with less bias, when compared to the distribution with no adjustment. Conclusion: These results can be used to estimate habitual energy and nutrient intake for adolescent population when it is only a single dietary measurement.

Keywords: Nutrients. Adolescents. Diet surveys. Nutrition assessment. Energy consumption. Methods.

\section{Introdução}

Estudos de prevalência de inadequação da ingestão de nutrientes são informativos para saúde pública, disponibilizando dados úteis para o diagnóstico nutricional, estabelecimento e monitoramento de políticas públicas em nutrição ${ }^{1}$.

Para coleta da ingestão de nutrientes a ser utilizada no cálculo da inadequação da ingestão, recomenda-se o uso de inquéritos dietéticos, como recordatórios de 24 horas (R24h) ou registro alimentar, por captarem o consumo com maior detalhamento das porções e formas de preparo dos alimentos consumidos ${ }^{1}$. No entanto, quando aplicados em um ou poucos dias, estes instrumentos falham ao captar as variações do dia-a-dia do consumo. Como consequência, a distribuição da ingestão se apresenta inflada, tendo efeito direto sobre a análise e a interpretação dos resultados. Um exemplo é a sub ou superestimativa da proporção de indivíduos abaixo ou acima de um dado critério de adequação ${ }^{2}$. O efeito da variação do dia-a-dia é reduzido com o aumento do número de R24h aplicados em cada indivíduo da população de estudo ${ }^{3}$. Em estudos epidemiológicos, entretanto, a coleta de múltiplos R24h geralmente é inviável, tanto em função de custos como da disponibilidade do indivíduo em relatar o seu consumo detalhadamente em várias ocasiões.

Uma alternativa é a utilização de métodos estatísticos para correção dos dados pela variabilidade intrapessoal do consumo ${ }^{4}$. Idealmente, esta correção é feita quando há pelo menos uma repetição do inquérito dietético em pelo menos uma subamostra representativa da população de estudo. Quando é aplicado somente um R24h para cada participante, a correção da distribuição da ingestão é possível utilizando-se variância intrapessoal externa, desde que obtida de população semelhante. Esta estratégia é recomendada ${ }^{5,6}$ e já foi utilizada na correção da distribuição da ingestão de nutrientes em adolescentes do município de São Paulo? .

Atualmente estão publicados componentes de variância intrapessoal para 
correção da distribuição da ingestão energia e nutrientes, obtidos de estudos brasileiros, referentes somente à população de adultos e idosos ${ }^{8}$. O objetivo deste estudo é disponibilizar componentes de variância intrapessoal para correção da distribuição da ingestão de nutrientes em adolescentes e, além disso, fornecer um exemplo da sua aplicação na correção da distribuição da ingestão de nutrientes proveniente de adolescentes de outro estudo.

\section{Métodos}

\section{População de estudo}

Para estimativa da variância intrapessoal da ingestão de nutrientes, foram utilizados dados do Inquérito de Saúde de São Paulo (ISA-Capital). Trata-se de um estudo transversal de base populacional que ocorre periodicamente no município de São Paulo. Foi utilizado processo de amostragem complexa, estratificada e por conglomerados, cujos detalhes são obtidos em outra publicação ${ }^{9}$. No ISA-Capital, coletado em 2003, foram entrevistados 813 adolescentes, que foram convidados a participarem do presente estudo, em 2007. Deste total, 314 permaneceram na faixa etária de 14 a 18 anos, dos quais $3 \%(n=9)$ se recusaram a participar, $15 \%(n=46)$ mudaram de endereço e não foram localizados, e $14 \%$ ( $\mathrm{n}=$ 44) não foram encontrados em casa mesmo após 3 visitas em horários e dias da semana distintos. Esta faixa etária é coincidente com o proposto pelo Instituto de Medicina como uma das fases da adolescência para a qual foram estabelecidas recomendações de ingestão de nutrientes. A amostra final foi de 215 adolescentes, sendo 112 do sexo masculino e 103 do sexo feminino.

Para ilustrar o efeito da aplicação dos componentes de variância intrapessoal, obtidos no presente estudo, na correção da distribuição da ingestão de nutrientes em uma população semelhante, foi utilizada uma amostra de adolescentes do sexo masculino que responderam a dois recordatórios de 24 horas (R24h) $(n=102)$, participantes do
Inquérito de Saúde de São Paulo, coletado entre 2008 e 2009. O processo de amostragem do ISA 2008 foi semelhante ao ISA 2003, com amostragem complexa, cujos detalhes são encontrados em outra publicação ${ }^{10}$.

\section{Coleta dos dados}

As entrevistas foram conduzidas nos domicílios, durante os anos de 2007 e 2008, por entrevistadores previamente treinados. O consumo alimentar foi coletado por meio de R24h utilizando-se o método passo-a- passo ${ }^{11}$. Para o cálculo da variância intrapessoal do consumo, todos os adolescentes $(\mathrm{n}=215)$ foram convidados a responder a um novo R24h, após um intervalo de aproximadamente dois meses. Esta coleta foi realizada por telefone e a taxa de resposta foi de $65 \%$ $(\mathrm{n}=73)$ e $70 \%(\mathrm{n}=73)$ para o sexo masculino e feminino, respectivamente. As coletas, tanto domiciliar quanto por telefone, foram realizadas de forma a cobrir todos os dias da semana e meses do ano. Anteriormente à digitação dos dados de consumo alimentar, as informações contidas em cada uma das coletas foram checadas com objetivo de monitorar a qualidade das entrevistas e definir a padronização para quantidades dos alimentos e receitas das preparações relatadas.

\section{Análise dos dados}

O consumo relatado foi convertido em valores de energia e nutrientes utilizando-se o software Nutrition Data System for Research (NDS, versão 2007, Nutrition Coordinating Center, University of Minnesota, Minneapolis). Foram consideradas, para o ferro e o folato, a fortificação mandatória em farinhas de trigo e milho, vigente no Brasil a partir 2004.

Os componentes de variância intrapessoal da distribuição da ingestão de energia, macro e micronutrientes foram calculados pelo método desenvolvido pela Iowa State University (ISU) ${ }^{3}$ utilizando o Software for Intake Distribution Estimation (PC-SIDE, versão 1.0, 2003; Department of Statistics, Iowa State University, Ames). Basicamente, 
o método é composto pelos seguintes procedimentos: ajustes preliminares pelo dia da semana e estação do ano e incorporação dos pesos amostrais; transformação para normalidade, por meio de transformação logaritma ou power; e estimativa dos componentes de variância intra e interpessoal por meio de um modelo de medida de erro.

Foi feito um modelo para cada nutriente, no qual foi incluído o dia e o mês da coleta, $\mathrm{e}$ variáveis de ponderação (unidade primária de amostragem, estrato e peso amostral). Os dados foram analisados separadamente para o sexo masculino e feminino. Para cada nutriente foram geradas medidas descritivas do consumo (média, desvio-padrão, erro-padrão e percentis) e os componentes de variância inter e intrapessoal. Outro parâmetro necessário na correção de dados dietéticos, o quarto momento, medida referente ao achatamento da distribuição (curtose), também foi calculado.

\section{Aplicação dos componentes de variância}

Utilizando-se o método ISU, a distribuição da ingestão de vitamina B6, baseada apenas no primeiro R24h respondido por cada adolescente, foi corrigida mediante $o$ componente de variância intrapessoal e o quarto momento da distribuição obtidos no presente estudo, referente ao sexo masculino. Para verificar o efeito da correção, foram estimadas distribuições de outras duas formas: utilizando-se apenas o primeiro R24h para cada adolescente sem qualquer correção; e utilizando-se o componente de variância intrapessoal obtida pela repetição da coleta na própria amostra. Para cada distribuição foi construído um gráfico de densidade de freqüência e também calculadas a média, o desvio padrão, os percentis de consumo e a prevalência de inadequação, ou seja, a proporção de indivíduos com ingestão abaixo do valor da necessidade média estimada ${ }^{1}$.

\section{Resultados}

Dezesseis por cento dos adolescentes apresentaram sobrepeso, $67 \%$ eram etilistas,
$11 \%$ tabagistas e $87 \%$ pertenciam a famílias cujo chefe tinha concluído no máximo 8 anos de estudo. Não houve diferença entre os sexos para estas variáveis. A Tabela 1 traz os componentes de variância intrapessoal e o quarto momento da distribuição da ingestão de energia e nutrientes para o sexo masculino e feminino.

A Tabela 2 mostra a média, o desvio padrão, os percentis de consumo e a prevalência de inadequação da ingestão de vitamina B6 na amostra de adolescentes do sexo masculino, calculados a partir da distribuição sem correção, corrigida pela própria variância intrapessoal e corrigida por variância externa. O efeito da correção com variância externa é notável, quando se compara à distribuição corrigida com a própria variância intrapessoal. Os desvios-padrão e os percentis foram semelhantes, bem como as prevalências de inadequação estimadas: $6 \%$ e $10 \%$, respectivamente; ambos substancialmente inferiores à inadequação observada quando utilizado somente um R24h (30\%). As distribuições estimadas são mais bem visualizadas na Figura 1.

\section{Discussão}

O presente estudo apresentou componentes de variância intrapessoal da ingestão de energia e nutrientes obtidos em uma amostra de adolescentes do município de São Paulo. Estes dados podem ser utilizados na estimativa da ingestão habitual de energia e nutrientes, em uma população semelhante, quando há disponível somente um R24h para cada indivíduo do estudo.

O efeito da correção pela variabilidade intrapessoal do consumo é notável, como está mostrado na Figura 1, e extensamente descrito na literatura ${ }^{4}$. Utilizando-se a variância externa foi possível obter um percentual de adolescentes com consumo abaixo do recomendado similar ao obtido quando corrigido pela variabilidade intrapessoal da própria amostra. Ambos foram aproximadamente $1 / 3$ do percentual quando utilizado somente um R24h. No exemplo foi utilizada 
Tabela 1 - Componentes de variância intrapessoal e quarto momento da distribuição da ingestão de energia e nutrientes entre adolescentes. São Paulo, 2007-2008.

Table 1 - Within-person component variance and forth moment of the energy and nutrient intake distribution in adolescents. São Paulo, 2007-2008.

\begin{tabular}{lcccc}
\hline & \multicolumn{2}{c}{ Masculino } & \multicolumn{2}{c}{ Feminino } \\
\cline { 2 - 5 } & $\begin{array}{c}\text { Comp. Var. } \\
\text { intrapessoal }\end{array}$ & $4^{\circ}$ momento & $\begin{array}{c}\text { Comp. Var. } \\
\text { intrapessoal }\end{array}$ & 40 momento \\
\hline Energia & 0,7846 & 3,67 & 0,5533 & 3,61 \\
Carboidrato & 0,7483 & 4,48 & 0,6061 & 3,19 \\
Gordura total & 0,8294 & 2,59 & 0,6058 & 3,21 \\
Gordura saturada & 0,8159 & 2,68 & 0,5841 & 3,35 \\
Gord. Mono-insaturada & 0,8244 & 2,06 & 0,5427 & 4,19 \\
Gord. Poli-insaturada & 0,9145 & 3,00 & 0,7157 & 3,13 \\
Gord. Trans & 0,8701 & 3,39 & 0,7446 & 3,41 \\
Proteína & 0,5581 & 2,78 & 0,7559 & 3,71 \\
Vitamina A & 0,7402 & 2,34 & 0,6045 & 5,70 \\
Vitamina D & 0,5583 & 2,86 & 0,5976 & 3,03 \\
Vitamina E & 0,8372 & 3,40 & 0,5360 & 3,60 \\
Vitamina K & 0,6654 & 2,46 & 0,6244 & 2,75 \\
Vitamina C & 0,8045 & 2,46 & 0,7101 & 2,82 \\
Tiamina & 0,7886 & 2,61 & 0,6224 & 3,04 \\
Riboflavina & 0,7325 & 3,24 & 0,6792 & 3,05 \\
Vitamina B6 & 0,7338 & 2,89 & 0,5725 & 2,36 \\
Vitamina B12 & 0,5921 & 3,17 & 0,7185 & 2,45 \\
Cálcio & 0,6208 & 3,34 & 0,6545 & 4,24 \\
Fósforo & 0,6479 & 2,84 & 0,7470 & 3,91 \\
Magnésio & 0,7052 & 2,81 & 0,5097 & 1,94 \\
Ferro & 0,7412 & 2,76 & 0,6912 & 2,62 \\
Zinco & 0,6353 & 3,27 & 0,7023 & 2,65 \\
Manganês & 0,9168 & 3,15 & 0,7532 & 2,38 \\
Cobre & 0,6549 & 3,75 & 0,6683 & 4,64 \\
Selênio & 0,8117 & 3,70 & 0,7283 & 3,23 \\
Sódio & 0,7046 & 4,22 & 0,6632 & 3,04 \\
Potássio & 0,8067 & 2,31 & 0,7428 & 4,21 \\
Folato & 0,9681 & 2,97 & 0,7736 & 3,77 \\
Niacina & 0,6482 & 3,03 & 0,7809 & 3,40 \\
Colesterol & 0,7347 & 3,33 & 0,7762 & 2,83 \\
Fibra & 0,6190 & 2,97 & 0,6263 & 2,63 \\
Ác. Pantontênico & 3,71 & 0,6954 & 3,23 \\
Ácido linoléico & 2,37 & 0,7324 & 3,24 \\
Ácido linolênico & 2,48 & 0,5588 & 3,87 \\
\hline
\end{tabular}

uma amostra de adolescentes similar àquela utilizada no cálculo dos componentes de variância intrapessoal. A princípio, sua utilização em uma amostra com características distintas (p. ex., adolescentes de outro município) poderia introduzir um viés nos resultados. Esta questão foi discutida no estudo de Jahns et al. (2005) ${ }^{5}$, que compararam a prevalência de inadequação da ingestão de vitamina $\mathrm{C}$ em uma amostra de norte-americanos utilizando-se componentes de variância obtidos de dois estudos, um norte-americano e um russo. Os autores concluíram que a utilização de variância externa é recomendada mesmo quando não se trata da mesma população, uma vez que 
Tabela 2 - Média, desvio padrão (dp), percentis de consumo e prevalência de inadequação da ingestão de vitamina B6 entre adolescentes do sexo masculino. São Paulo, 2008-2009.

Table 2 - Mean, standard deviation, percentiles of intake and prevalence of inadequate intake of vitamin B6 among male adolescents. São Paulo, 2008-2009.

\begin{tabular}{lccccccc}
\hline & Média (dp) & \multicolumn{7}{c}{ percentis de consumo } & \multirow{2}{*}{ inad (\%) } \\
\cline { 3 - 6 } & & 10 & 25 & 50 & 75 & 90 & \\
\hline Sem correção & $1,80(1,09)$ & 0,76 & 1,06 & 1,56 & 2,13 & 2,96 & 30 \\
Corr. var 2009 $^{\mathrm{b}}$ & $1,79(0,59)$ & 1,10 & 1,37 & 1,73 & 2,15 & 2,59 & 10 \\
Corr. Var 2007c & $1,80(0,52)$ & 1,19 & 1,42 & 1,73 & 2,10 & 2,50 & 6 \\
\hline
\end{tabular}

a utilizando-se apenas o primeiro recordatório de 24 horas para cada participante;

${ }^{\text {b }}$ distribuição corrigida pela variância intrapessoal obtida da repetição do inquérito na própria amostra;

' distribuição utilizando primeiro recordatório de 24 horas para cada participante, corrigida pela variância externa;

${ }^{\mathrm{d}}$ prevalência de inadequação da ingestão.

a based on only a single 24-hour recall for each participant;

${ }^{b}$ distribution adjusted for the within-person variance from the replicate of the 24-hour recall in the same sample;

' distribution based on only a single 24-hour recall but adjusted for the external within-person variance;

${ }^{d}$ prevalence of inadequate nutrient intake.
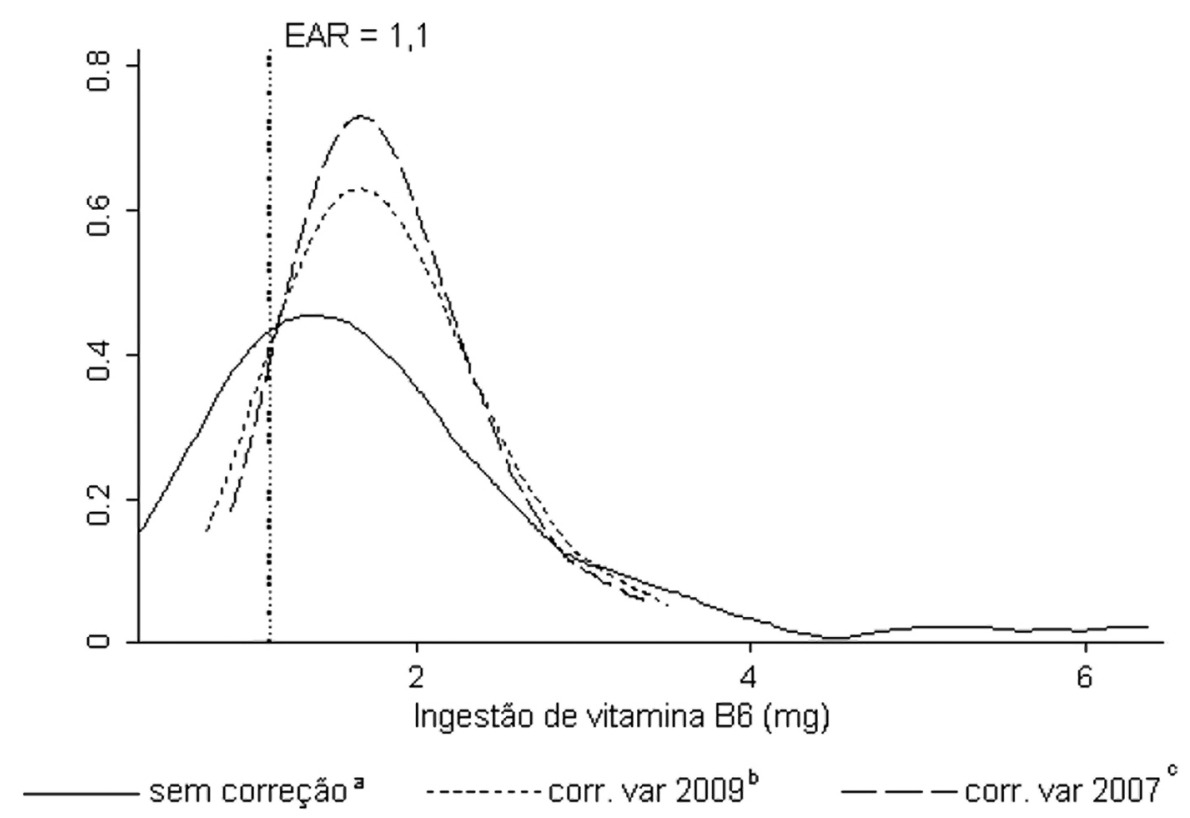

a utilizando-se apenas o primeiro recordatório de 24 horas para cada participante;

${ }^{\text {b }}$ distribuição corrigida pela variância intrapessoal obtida da repetição do inquérito na própria amostra;

' distribuição utilizando primeiro recordatório de 24 horas para cada participante, corrigida pela variância externa;

a based on only a single 24-hour recall for each participant;

${ }^{b}$ distribution adjusted for the within-person variance from the replicate of the 24-hour recall in the same sample;

' distribution based on only a single 24-hour recall but adjusted for the external within-person variance

Figura 1 - Distribuição da ingestão de vitamina B6 entre adolescentes do sexo masculino. São Paulo, 2008-2009.

Figure 1 - Distribution of intake of vitamin B6 among male adolescents. São Paulo, 2008-2009.

o pior cenário é a obtenção da distribuição em apenas um R24h.

A correção dos dados por componentes de variância externos envolve uma série de procedimentos estatísticos, o que a torna inviável sem o auxílio do software PC-SIDE. Este software não é gratuito. No entanto, a Organização Mundial da Saúde (OMS) está desenvolvendo um software de domínio público, baseado na mesma metodologia. Este, 
entre outras finalidades, possibilita corrigir a distribuição do consumo de nutrientes utilizando componentes de variância externos. O desenvolvimento do software faz parte do Intake, Monitoring, Assessment and Planning Program (IMAPP) da OMS, cujo documento com detalhes se encontra disponível na internet*.

O componente de variância intrapessoal representa o percentual da variância total que é explicado pela variação do dia-a-dia para o conjunto de indivíduos de uma população. Quanto maior o valor, maior é a variação da quantidade ingerida de um dia para o outro relativa a um mesmo nutriente. Este componente tem sido descrito como a principal fonte de variação da ingestão de nutrientes ${ }^{9}$ e varia de acordo com a população, faixa etária e sexo estudado.

A diferença entre os sexos é mais bem evidenciada em relação à energia e aos macronutrientes. Entre o sexo masculino, elevados componentes de variância intrapessoal foram observados para energia, carboidratos e lipídio; e baixos para proteína. Entre o sexo feminino, observou-se o contrário: valores menores para energia, carboidrato e lipídio, e elevado para proteína. Isto sugere que a ingestão calórica é mais estável entre as meninas, ao passo que varia em maior magnitude de um dia para o outro entre adolescentes do sexo masculino.

O método ISU, bem como demais métodos disponíveis, gera percentis da distribuição da ingestão habitual para grupos de indivíduos, e não valores de ingestão habitual de cada indivíduo da amostra. Isto torna limitadas as formas de análises que associam a ingestão habitual estimada com múltiplas covariáveis. Testes de diferença de médias e de proporção podem ser calculados estimando-se uma distribuição para cada categoria do grupo que se deseja comparar. Por exemplo, pode-se testar se homens e mulheres diferem estatisticamente em relação ao seu consumo de um dado nutriente. Primeiramente, estima-se a ingestão habitual para cada sexo. O teste $t$ é calculado utilizando-se valores de média e desvio-padrão estimados para cada grupo.

A amostra utilizada no presente estudo foi baseada em uma amostra representativa da população de adolescentes do município de São Paulo. Porém, em função do grande número de indivíduos que deixaram de ser adolescentes, desde o sorteio da amostra inicial, em 2003, até o retorno aos domicílios para nova coleta de dados, em 2007, a amostra pode ter perdido sua característica de representatividade. No entanto, dos 59 setores censitários utilizados na amostra inicial, 53 continuaram contemplados, representando, da mesma forma, as diversas regiões do município de São Paulo. Além disso, não houve diferença estatística entre os estratos (de acordo com o percentual de chefe da família com nível universitário) obtidos na amostra de 2003 e 2007.

Concluindo, os componentes de variância intrapessoal podem ser empregados em populações semelhantes à amostra do estudo para se estimar a ingestão habitual de energia e nutrientes quando há disponível somente um R24h para cada participante do estudo.

*Disponível em: http://www.who.int/entity/nutrition/publications/micronutrients/9789241599603.pdf

\section{Referências}

1. Institute of Medicine, Food and Nutrition Board. Dietary Reference Intakes: Applications in Dietary Assessment. Washington, DC: National Academy Press; 2000.

2. Beaton GH. Approaches to analysis of dietary data: relationship between planned analyses and choice of methodology. Am J Clin Nutr 1994; 59(S): 253-61.
3. Nusser SM, Carriquiry AL, Dodd KW, Fuller WA. A semiparametric transformation approach to estimating usual daily intake distributions. J Am Stat Assoc 1996; 91: 1440-9.

4. Dodd KW, Guenter PM, Freedman LS, Subar AF, Kipnis V, Midthune D et al. Statistical Methods for Estimating Usual Intake of Nutrients and Foods: A Review of the Theory. J Am Diet Assoc 2006; 106: 1640-50. 
5. Jahns L, Arabe L, Carriquiry A, Popkin BM. The use of external whitin-person variance estimates to adjust nutrient intake distributions over time and across population. Public Health Nutr 2005; 8: 69-76.

6. Murphy SP, Guenther PM, Kretsch MJ. Using the dietary reference intakes to assess intakes of groups: pitfalls to avoid. J Am Diet Assoc 2006; 106: 1550-3.

7. Verly-Jr E, Cesar CLG, Fisberg RM, Marchioni DML. Socio-economic variables influence the prevalence of inadequate nutrient intake in Brazilian adolescents: results from a population-based survey. Public Health Nutr 2011; 14: 1533-8.

8. Morimoto JM, Marchioni DML, Cesar CLG, Fisberg RM. Variância intrapessoal para ajuste da distribuição de nutrientes em estudos epidemiológicos. Rev Saúde Pública 2011; 45: 621-5.
9. Verly-Jr E, Cesar CLG, Fisberg RM, Marchioni DML. Source of variation of energy and nutrient intake among adolescents in São Paulo. Cad Saúde Pública 2010; 26: 2129-37.

10. Bigio RS. Consumo de frutas, legumes e verduras: relação com níveis sanguíneos de homocisteína entre adolescentes [dissertação de mestrado]. São Paulo: Faculdade de Saúde Pública da Universidade de São Paulo; 2011.

11. Thompson FE, Byers T. Dietary assessment resource manual. J Nutr 1994; 124(Suppl): 2245-317.

Recebido em: 22/11/10

Versão final apresentada em: 20/10/11

Aprovado em: 10/02/12 